\title{
Two-state migration of DNA in a structured microchannel
}

\author{
Martin Streek, ${ }^{1}$ Friederike Schmid, ${ }^{1}$ Thanh Tu Duong, ${ }^{2}$ Dario Anselmetti, ${ }^{2}$ and Alexandra Ros ${ }^{2}$ \\ ${ }^{1}$ Kondensierte Materie, Universität Bielefeld, Fakultät für Physik, D-33615 Bielefeld, Germany \\ ${ }^{2}$ Experimentelle Biophysik \& Angewandte Nanowissenschaften, Universität Bielefeld, Fakultät für Physik, D-33615 Bielefeld, Germany
}

(Received 19 August 2004; published 19 January 2005)

DNA migration in topologically structured microchannels with periodic cavities is investigated experimentally and with Brownian dynamics simulations of a simple bead-spring model. The results are in very good agreement with one another. In particular, the experimentally observed migration order of $\lambda$ - and T2-DNA molecules is reproduced by the simulations. The simulation data indicate that the mobility may depend on the chain length in a nonmonotonic way at high electric fields. This is found to be the signature of a nonequilibrium bistability between two different migration states, a slow one and a fast one. The latter can also be observed experimentally under appropriate conditions.

\section{INTRODUCTION}

DNA electrophoresis is one of the main techniques to separate DNA molecules by size [1]. Since the mobility of DNA molecules does not depend on the chain length in free solution, electrophoresis is usually performed in gels. Given the progress of microtechnology, much effort has been spent on the integration of DNA electrophoresis into microfluidic devices (lab-on-a-chip devices) [2] and separation strategies which rely on well-defined microscopic structures have been explored. Various such gel-free devices exploiting both dc [3-7] and ac [8,9] fields have been proposed. The mobility of the DNA in these microstructures is determined by a subtle interplay of the characteristic dimensions of the microstructure and the molecular size. Microstructured systems allow for fast separation of kbp size DNA fragments in timescales less than one minute [8-10].

In the present work, we focus on a geometry which has been investigated by Han and Craighead et al. $[6,11,12]$ and later by us (Duong et al. [7]). The DNA is driven through a periodic sequence of cavities and constrictions (Fig. 1) with an electrical dc field. The separation experiments of Han and Craighead motivated several computer simulation studies [13-15], which reproduced the results and lead to an improved understanding of the DNA separation mechanisms in these channels. Originally $[8,11]$, the width of the constriction was chosen of the order of $0.1 \mu \mathrm{m}$ (corresponding to a few persistence lengths of DNA molecules), and entropic trapping at the entrances of the constrictions was proposed to be the main mechanism leading to size-dependent migration of DNA in these channels. Recently, an additional effect that relies on diffusion has been revealed in such geometries [15]. This second mechanism does not premise extremely narrow constrictions. Therefore, one should also achieve DNA separation with wider channels.

Based on that prediction, we have investigated experimentally the size-dependent electrophoresis in structured microchannels (Fig. 1) with constriction widths corresponding roughly to the radius of gyration of the tested DNA molecules $[7,10]$. We found that separation by size is possible [10]. Unexpectedly, however, the relation between mobility and chain length turned out to be inverse to that observed by
Han and Craighead: the short $\lambda$-DNA (48 kbp) migrated faster than the longer T2-DNA (164 kbp).

In the present paper, we explore the reasons for this unforeseen behavior by Brownian dynamics simulations of a simple bead-spring model. Models of this kind have been used successfully in the past to study the migration of DNA in various geometries [15-19]. Our simulations reveal a surprisingly complex phenomenology. If the model parameters are adjusted to those of the experiment, we reproduce the experimental findings. With other model parameters (smaller structures, lower electric field), we recover the originally expected increase of mobility with increasing chain length. As we show in this paper, the reversal of this trend turns out to be the signature of a nonequilibrium bistability, which emerges at even higher fields or in even larger structures.

a)
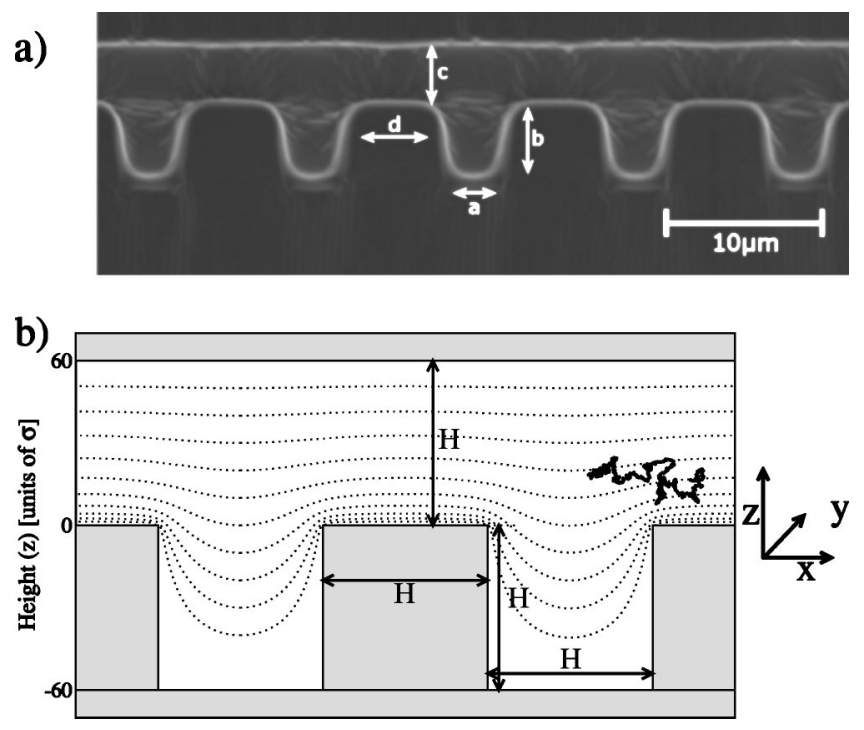

FIG. 1. (a) SEM image of an experimental device presented by Duong et al. [7], and (b) schematic of the channels. As all size parameters are equal, only one parameter $H$ is necessary to describe the structure. The coordinate system used in the simulations is given on the right. The dotted lines represent the electric field lines. Also shown in (b) is a configuration snapshot from a simulation of a chain with $N=320$ monomers. 
However, unfavorable for separation in specified geometries, this prediction implies the existence of two migration states, which could indeed be observed in migration experiments.

The paper is organized as follows. The simulation model and the simulation technique are described in Sec. II, and the experimental setup in Sec. III. In Sec. IV, we briefly discuss the simulations corresponding to the earlier experimental study by Duong et al. [7]. The results on the two coexisting migration states are presented in Sec. $\mathrm{V}$, and the possible transition mechanisms are discussed in Sec. VI. We summarize and conclude in Sec. VII.

\section{THE SIMULATION MODEL}

\section{A. Definition of the model}

We describe the DNA on a coarse-grained level by a bead-spring model. Several base pairs of DNA are represented by one bead or "monomer." Neighboring beads are connected by harmonic springs

$$
V_{\mathrm{sp}}(r)=\frac{k}{2} r^{2},
$$

where $r$ is the distance between the two neighboring monomers. All beads interact with a purely repulsive WeeksChandler-Andersen (WCA) potential

$$
V_{\text {pair }}(r) / k_{\mathrm{B}} T= \begin{cases}\left(\frac{\sigma}{r}\right)^{12}-\left(\frac{\sigma}{r}\right)^{6}+\frac{1}{4}, & \left(\frac{r}{\sigma}\right) \leqslant 2^{1 / 6}, \\ 0, & \text { otherwise, }\end{cases}
$$

where $r$ is the distance between two monomers and $\sigma \equiv 1$ sets the range of the interaction.

The walls are assumed to be soft and purely repulsive. The potential describing the interaction with the walls is the same as that acting between the monomers [Eq. (2), with $r$ as the the minimum distance between a bead and the wall]. In the corners, the forces of the adjacent walls are summed up. To model the finite depth of the device, we also introduce walls in $y$ direction.

Each bead carries a charge $q$ and is subject to an electrical force $\vec{f}_{\mathrm{el}}=q \vec{E}=-q \nabla \Phi$, but charges do not interact with one another in our model. The field is calculated by solving the Laplace equation $(\Delta \Phi=0)$ in the channel. We use von Neumann boundary conditions at the walls $(\vec{n} \cdot \nabla \phi=0$, with $\vec{n}$ the surface normal) and impose a constant potential difference between the inlets of the device. A numerical solution $\Phi(\vec{r})$ is obtained from the software program MATLAB (Mathworks, U.S.), using a finite element solver. The grid values of the derivatives are interpolated bilinearly. The field lines are shown in Fig. 1. Throughout the rest of this paper, the field denoted by $E$ refers to the total potential difference divided by the total channel length, and can thus be regarded as the characteristic strength of the electric field.

The motion of the chain is described by Langevin dynamics, i.e., the solvent surrounding the chain is replaced by a Brownian force $\vec{\eta}_{i}$ and an effective friction coefficient $\zeta \equiv 1$, which fulfill the conditions [20]

$$
\left\langle\eta_{i}\right\rangle=0,
$$

$$
\left\langle\eta_{i, \alpha}(t) \eta_{j, \beta}\left(t^{\prime}\right)\right\rangle=2 \zeta k_{\mathrm{B}} T \delta_{i j} \delta_{\alpha \beta} \delta\left(t-t^{\prime}\right),
$$

with monomer indices $i, j=1 \ldots N$, Cartesian directions $\alpha, \beta$ $\in\{x, y, z\}$ and $t, t^{\prime}$ two given times. The equations of motion are thus

$$
\begin{gathered}
\dot{\vec{r}}_{i}=\vec{v}_{i}, \\
m \dot{\vec{v}}_{i}=\vec{f}_{i}-\zeta \vec{v}_{i}+\vec{\eta}_{i},
\end{gathered}
$$

where $\left\{\vec{r}_{i}\right\}$ and $\left\{\vec{v}_{i}\right\}$ represent the locations and the velocities of the monomers, respectively. The vectors $\left\{\vec{f}_{i}\right\}$ sum up forces acting on a monomer (harmonic spring, repulsive monomer interaction, wall interaction, external electric field). Here, each bead carries a mass $m$, but simulations with vanishing mass were also carried out for comparison (see Sec. VI). With $m=0$, the equations of motion reduce to

$$
\zeta \dot{\vec{r}}_{i}=\vec{f}_{i}+\vec{\eta}_{i}
$$

The natural units of our simulation are defined in terms of the bead size $\sigma$, the friction coefficient $\zeta$, the bead charge $|q|$, and the temperature $T$. Based on these quantities, the energy unit is $k_{\mathrm{B}} T$, the length unit is $\sigma$, the time unit is $t_{0}$ $=\zeta \sigma^{2} / k_{\mathrm{B}} T$, and the electric field unit is $E_{0}=k_{\mathrm{B}} T / \sigma|q|$.

The dynamical equations [Eqs. (5) and (6)] are integrated with a Verlet algorithm using a time step $\Delta_{\mathrm{t}}=10^{-2} t_{0}$. In the case of the relaxational dynamics in Eq. (7), we use a Euler algorithm with the time step $\Delta_{\mathrm{t}}=10^{-4} t_{0}$. The stochastic noise $\vec{\eta}_{i}$ was implemented by picking random numbers at every time step. As shown in Ref. [21], the random numbers do not have to be Gaussian distributed, as long as they fulfill Eqs. (3) and (4). Those used in our simulation were evenly distributed inside the unit sphere. Unless stated otherwise, the run lengths were $(4-20) \times 10^{8} \Delta_{t}$ with equilibration times of $2 \times 10^{7} \Delta_{\mathrm{t}}$.

The spring constant in Eq. (1) was chosen very high, $k$ $=100 k_{\mathrm{B}} T / \sigma^{2}$. This ensures that no chain crossing occurs [22]. The equilibrium length of each bond is $0.847 \sigma$.

The static properties of free chains of length $N$ correspond to those of self-avoiding random walks [22] with the persistence length $l_{\mathrm{p}}=1.6 \sigma$ and the radius of gyration $R_{\mathrm{g}}$ $\approx 0.5 \sigma N^{\nu}$. Here $\nu=0.588$ is the Flory exponent. The dynamical properties are characterized by the diffusion constant $D$ $=k_{\mathrm{B}} T / N \zeta=N^{-1} \sigma^{2} / t_{0}$, the mobility $\mu_{0}=|q| / \zeta=\sigma / E_{0} t_{0}$, which is independent of the chain length $N$, and the decay time of the drift velocity $\tau_{0}=m / \zeta$ [Eq. (6)]. The quantity $\tau_{0}$ sets the timescale on which inertia effects are significant. In most simulations presented here, it was set to $\tau_{0}=t_{0}$ (i.e., $m=\zeta t_{0}$ ), based on the assumption that the relevant timescales for the dynamical processes of interest here are much longer. In some cases, however, simulations with vanishing $\tau_{0}$ (vanishing mass) were also necessary (see Sec. VI). On timescales larger than $\tau_{0}$, the chains behave like standard (self-avoiding) Rouse chains [20] with the Rouse relaxation time $\tau_{\mathrm{R}}$ $\approx 0.045 t_{0} N^{1+2 \nu}$.

\section{B. Correspondence between the model and the experiment}

In the experiments, the mobility of $\lambda$-DNA (48 kbp) and T2-DNA (164 kbp) in microchannels with geometries such 
TABLE I. Adaptation of the simulation units to various channel sizes. All values have been rounded to two digits.

\begin{tabular}{lccc}
\hline \hline & $H=1.5 \mu \mathrm{m}$ & $H=3 \mu \mathrm{m}$ & $H=5 \mu \mathrm{m}$ \\
\cline { 2 - 4 } $1 \mathrm{bead}$ & $48 \mathrm{bp}$ & $150 \mathrm{bp}$ & $340 \mathrm{bp}$ \\
$\lambda$-DNA & 1000 beads & 330 beads & 140 beads \\
T2-DNA & 3500 beads & 1200 beads & 490 beads \\
$1 \mu \mathrm{m}$ & $40 \sigma$ & $20 \sigma$ & $12 \sigma$ \\
$1 \mathrm{~s}$ & $7.5 \times 10^{5} t_{0}$ & $6.2 \times 10^{4} t_{0}$ & $9.5 \times 10^{3} t_{0}$ \\
$1 \mathrm{~V} / \mathrm{cm}$ & $9.8 \times 10^{-5} E_{0}$ & $5.9 \times 10^{-4} E_{0}$ & $2.3 \times 10^{-3} E_{0}$ \\
$E_{0}$ & $10 \mathrm{kV} / \mathrm{cm}$ & $1.7 \mathrm{kV} / \mathrm{cm}$ & $430 \mathrm{~V} / \mathrm{cm}$ \\
\hline \hline
\end{tabular}

as sketched in Fig. 1 was studied. Details are given in Ref. [7] and Sec. III. We will now discuss how the parameters and units of our model can be related to those of the experiment.

The energy unit is simply given by the temperature, $k_{\mathrm{B}} T$ $=300 k_{\mathrm{B}}, K \approx 0.026 \mathrm{eV}$, at which the experiments were carried out. Next we adjust the value of the length unit. In our earlier work on entropic traps [15], we had matched the persistence lengths of the model chains with that of DNA. In the present case, however, the persistence length is not an experimentally relevant length scale, because all dimensions of the microchannels are on the order of magnitude of the radius of gyration. Thus we can determine the length unit by comparing the characteristic length $H$ in our model channel (see Fig. 1) to the corresponding experimental value. Note that this implies that we can relate simulations with one channel geometry to all three experimental channels by scaling our results accordingly.

More specifically, the simulations were carried out using channels with $H=60 \sigma$. We illustrate our adaptation with the example of the $5 \mu \mathrm{m}$ microchannels. It is carried out in four steps. First, we compare the channel height $H=5 \mu \mathrm{m} \equiv 60 \sigma$. This gives the length unit $1 \sigma \equiv 83 \mathrm{~nm}$. Second, comparing the radii of gyration of our model chains with that of $\lambda$-DNA, we find that $\lambda$-DNA is represented by chains of $N$ $=140$ beads, or 1 bead $\equiv 340$ bp. Third, the time unit is adjusted by matching the diffusion constant $D$ for $\lambda$-DNA. Experimentally, Smith et al. have reported $D$ $=0.47 \pm 0.03 \mu \mathrm{m}^{2} / \mathrm{s}$ [23]. Comparing this with the theoretical value, $D=\sigma^{2} / N t_{0}$, we obtain $t_{0} \equiv 1.1 \times 10^{-4} \mathrm{~s}$. Hence, one second corresponds to $9.5 \times 10^{3} t_{0}$ (or $\sim 10^{6}$ time steps). Finally, the electric field unit $E_{0}$ is adjusted by adjusting the mobility in large unstructured microchannels, which is chain length independent and given by $\mu_{0}=1.84 \times 10^{-4} \mathrm{~cm}^{2} / \mathrm{V} \mathrm{s}$ [7]. It is the sum of the free flow mobility $\mu_{0}$ and the electroosmotic mobility $\mu_{\text {eof }}=(2.9 \pm 0.6) \times 10^{-4} \mathrm{~cm}^{2} / \mathrm{V} \mathrm{s}$ in our channels (see below). This leads to $1 \mathrm{~V} / \mathrm{cm} \equiv 2.3 \times 10^{-3} E_{0}$. These values as well as those obtained for channels with $H$ $=3 \mu \mathrm{m}$ and $H=1.5 \mu \mathrm{m}$ are summarized in Table I. Unless stated otherwise, all adaptations given in this paper refer to the $5 \mu \mathrm{m}$ channels, except in Sec. IV.

We set the depth of the device to $60 \sigma$. For comparison, we also performed simulations with infinite depth of the device and found no qualitative difference (data not shown). In our case, the depth has no significant influence on the dynamics.
The model disregards a number of important physical effects.

First, electrostatic interactions are neglected. The Debye screening length of DNA in typical buffer solutions is about $2 \mathrm{~nm}$, which is comparable to the diameter of the polymer and much smaller than the persistence length of DNA (roughly $50 \mathrm{~nm}$ ).

Second, PDMS exhibits silanol groups on its surface which dissociate under the experimental conditions. Thus the experiments were carried out in microchannels with negatively charged surfaces. This implies the generation of cathodic electroosmotic flow, and the resulting mobility of DNA molecules is a sum of the electroosmotic and electrophoretic mobilities. The DNA molecules migrate to the anode, indicating that electrophoresis overcomes electroosmosis. In the simulation, we do not incorporate any electro-osmotic flow, or flow in general. However, recent reports $[24,25]$ show that for sufficiently low Reynolds number, the flow outside the Debye layer at the walls is rotation free and proportional to the electric field with a fixed proportionality constant $\alpha$. Let $\vec{v}_{0}(\vec{r})$ denote the flow field at a given position $\vec{r}$ and $\vec{f}_{\mathrm{o}, i}$ the forces acting on monomer $i$, excluding the electric force $\vec{f}_{\mathrm{el}, i}=-q \cdot \nabla \Phi\left(\vec{r}_{i}\right)$. Using $\vec{v}_{0}(\vec{r})=\alpha \cdot \nabla \Phi(\vec{r})$, the equation of motion [Eq. (6)] now becomes

$$
\begin{aligned}
m \dot{\vec{v}}_{i} & =\vec{f}_{\mathrm{o}, i}+\vec{f}_{\mathrm{el}, i}-\zeta\left(\vec{v}_{i}+\vec{v}_{0}(\vec{r})\right)+\eta_{i} \\
& =\vec{f}_{\mathrm{o}, i}-(q+\zeta \alpha) \cdot \nabla \Phi(\vec{r})+\eta_{i},
\end{aligned}
$$

and for the overdamped dynamics [Eq. (7)] one finds

$$
\zeta \dot{\vec{r}}_{i}=\vec{f}_{\mathrm{o}, i}-(q+\zeta \alpha) \cdot \nabla \Phi(\vec{r})+\eta_{i}
$$

These equations are formally equivalent to Eqs. (6) and (7) with a rescaled charge $q$. The Reynolds number for the electro-osmotic flow is easily found for our device. Using the viscosity of water, the electro-osmotic mobility given above and a characteristic length of $5 \mu \mathrm{m}$, the Reynolds number is roughly $10^{-3}$ for electric fields of $100 \mathrm{~V} / \mathrm{cm}$ in our channels. This justifies our adaptation to the overall mobility given above.

Third, hydrodynamic effects are not taken into account. This approximation must be questioned. On the one hand, DNA is always surrounded by counterions, which are dragged into the opposite direction of the DNA. Thus the DNA molecule experiences not only hydrodynamic drag, but also an extra friction from the solvent molecules. In free solution, these two effects cancel each other [1]. This "hydrodynamic screening" accounts for the free-draining property of DNA. However, the total cancellation fails if the DNA molecule is blocked by a geometric barrier [26]. In that case, the counterions will not be immobilized, since counterions are still free to move. Furthermore, hydrodynamic interactions affect the diffusion constant $D$. In our model, it scales the chain length $N$ like a Rouse chain $(D \propto 1 / N)$. Including hydrodynamic interactions, one would expect Zimm scaling $\left(D \propto 1 / R_{g} \propto 1 / N^{\nu}\right)$. In experiments, the diffusion constant of DNA is found to scale as $D \propto 1 / N^{0.672}$ [27].

Unfortunately, a full simulation which treats hydrodynamic interactions correctly, takes into account the dynami- 
a)

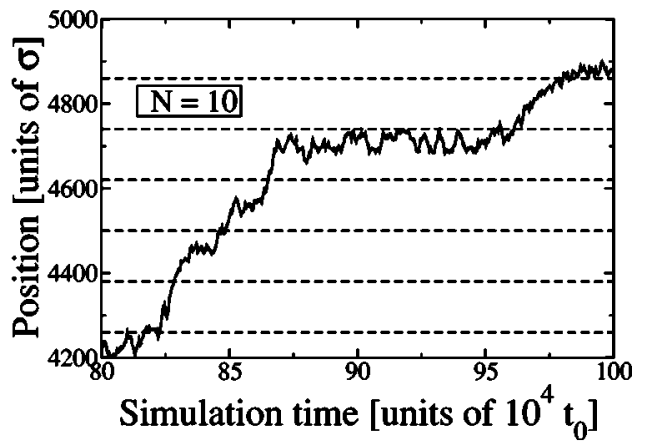

b)

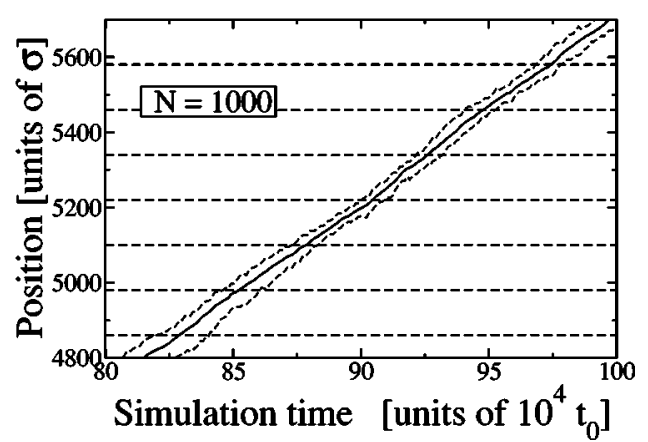

FIG. 2. Trajectories for chains of length (a) $N=10$, and (b) $N$ $=1000$, at $E=0.005 E_{0}$. The horizontal dashed lines mark the beginning of the narrow regions. The solid line in the middle shows the position of the center of mass; the dashed lines indicate the leading and trailing monomers. For $N=10$ (a), these lines cannot be distinguished from each other.

cal counter ion distribution, and includes electrostatic interactions, is a formidable task and impossible with standard computer resources. The simplifications described above will affect the quantitative results, but presumably neither by orders of magnitude nor qualitatively.

\section{EXPERIMENTAL SETUP}

The fabrication method used for the structured microchannels is based on soft lithography of poly(dimethylsiloxane) (PDMS) and is described in detail in Ref. [7]. Briefly, a photoresist (SU-8 from Microresist, GER) coated Si-wafer (CrysTec, GER) is exposed through a chromium mask (DeltaMask, NL). After developing and hard-baking liquid PDMS (Sylgard 184, Dow Corning, USA) is poured onto the master containing the inverted microstructure and baked for $3 \mathrm{~h}$ at $75^{\circ} \mathrm{C}$. Peeling off the PDMS slab, punching reservoir holes through the PDMS and covering it with a clean microscope glass slide results in the microchip. Figure 2(b) shows a scanning electron micrograph (SEM) image of the topview of a microchannel with $H=5 \mu \mathrm{m}$. In comparison to the simulated geometry, the corners of the cavities are rounded and the lengths $a, b, c$, and $d$ in Fig. 1 are not exactly equal. The exact values were $a=3.6 \mu \mathrm{m}, b=6.0 \mu \mathrm{m}, c=3.7 \mu \mathrm{m}$, and $d=4.7 \mu \mathrm{m}$. The depth of the microchannel was $2.8 \mu \mathrm{m}$. Other channel geometries mentioned in this work are described in detail in Ref. [7].

For fluorescence imaging, $6 \mathrm{pM} \lambda$ - or T2-DNA (Fluka, Germany) in the same buffer $[10 \mathrm{mM}$ Tris at $\mathrm{pH} 8.3$ contain- ing $50 \mathrm{mM} \mathrm{NaCl}, 1 \mathrm{mM}$ ethylenediaminetetraacetic acid (EDTA) and $2 \%(\mathrm{v} / \mathrm{v}) \beta$-Mercaptoethanol] were adjusted to a YOYO-1 (Molecular Probes, USA) in a base-pair ratio of 1:7.5. A sensitive fluorescence videomicroscopy setup is used to record DNA migration, as described in Ref. [7]. Briefly, it consists of an inverted microscope (Axivert 100, Zeiss, GER) with a filter set for fluorescence observation (XF100-3, Omega, USA), a $100 \times$ oil immersion objective (Plan Neofluar NA 1.3, Zeiss, GER) and a sensitive CCDcamera (Imager 3LS, LaVision, GER). Data acquisition and automated data analysis is performed in DaVis 5.4.4 (LaVision) using cross-correlation analysis. Electric fields are applied with power supplies from FUG (MCN 14-2000, GER).

The electro-osmotic mobility was determined according to the current monitoring method [28]. Linear channels of $20 \mu \mathrm{m}$ width and depth were filled with water immediately after assembly, which was exchanged by the $10 \mathrm{mM}$ sample buffer without DNA before the experiment. The buffer in the anode reservoir was then exchanged by $8 \mathrm{mM}$ Tris buffer with otherwise identical composition as the $10 \mathrm{mM}$ buffer. A constant electric field of $400 \mathrm{~V} / \mathrm{cm}$ was applied and the current was recorded until a stable lower current value was reached. The recorded current decay times $\tau_{\text {eof }}$ were used to calculate the electro-osmotic mobility according to $\mu_{\text {eof }}$ $=l / E \tau_{\text {eof }}$, with $l$ the length of the channel, and $E$ the applied electric field.

\section{LOW ELECTRIC FIELD: STEADY-STATE MIGRATION}

We begin by presenting simulations for relatively low electric fields $E$, which can be compared to the previously published experimental results of Duong et al. [7]. In this section, we will adjust our model units to the channels with characteristic size $H=3 \mu \mathrm{m}$ (cf. Sec. II B), where Duong et al. had found particular clear evidence of a size-dependent mobility.

The electric field was varied from $E=0.0025 E_{0}$ up to $E$ $=0.04 E_{0}$, which corresponds to experimental fields in the range of $4.2 \mathrm{~V} / \mathrm{cm}$ up to $67 \mathrm{~V} / \mathrm{cm}$. The chain lengths range from $N=10$ up to $N=2000$ monomers, modeling DNA strands from 1.5 up to $290 \mathrm{kbp}$. Figure 2 shows typical trajectories obtained at $E=0.005 E_{0}$. The trajectories are similar to those obtained for the entropic trap geometry [15]. For $N=10$, the trajectory is completely dominated by diffusion. Long chains with $N=1000$ monomers are hardly affected by the constrictions-much less, in fact, than in the case of the entropic traps [15]. This is because the width of the narrow regions is of the order of the radius of gyration; the chain is thus able to cross the constriction without uncoiling. The trapping mechanism suggested by Han et al. [6] should not apply here. The fact that some trapping nevertheless occurs for short chains underlines the importance of the effect reported by Streek et al. [15]. Indeed, Fig. 3 shows that the mobility increases as a function of the chain length for $E$ $=0.005 E_{0}$. In the experimental channel, $E=0.005 E_{0}$ corresponds to a real field of $E \sim 8.4 \mathrm{~V} / \mathrm{cm}$. In such low fields, Duong et al. [7] were unable to distinguish between different mobilities for different DNA sizes; the statistical error is 


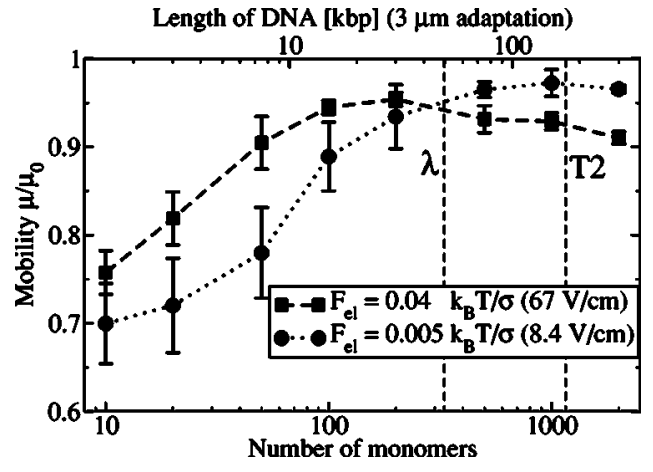

FIG. 3. Mobility as a function of chain length for $E=0.005 E_{0}$ $(8.4 \mathrm{~V} / \mathrm{cm})$ and $E=0.04 E_{0}(67 \mathrm{~V} / \mathrm{cm})$. The vertical dashed lines indicate the length of $\lambda$ - and T2-DNA, with adaptation for the $3 \mu \mathrm{m}$ constrictions. Experimentally, Duong et al. have reported $\mu / \mu_{0}$ $=0.99 \pm 0.1$ for $\lambda$-DNA and $\mu / \mu_{0}=0.63 \pm 0.03$ for T2-DNA [7].

large because diffusive motion becomes significant. We note that in simulations, we can track the DNA migration over many more cavities than in the experiments, therefore the relative error is smaller.

At higher fields, Duong et al. [7] reported the inverse effect-the mobility of T2-DNA is smaller than that of $\lambda$-DNA. If we increase the electric field in the simulations accordingly, we find indeed that, at $E=0.04 E_{0}$, the mobility reaches a maximum at $N=200$ beads $(29 \mathrm{kbp})$ and decreases for longer chains (Fig. 3). In the parameter region corresponding to $\lambda$-DNA and T2-DNA, smaller chains migrate faster than longer ones, but the difference in the mobility is not as pronounced as in experiment. Thus our simulations reproduce the behavior observed by Duong et al. [7] qualitatively.

This result is gratifying, but it does not yet explain why the chain length dependence of the mobility is suddenly reversed. In order to explore this question, we proceeded to investigate the migration in our structures under more extreme conditions. In the simulations, we can increase the field strength $E$. In the experiments, this can only be realized up to a field of approximately $100 \mathrm{~V} / \mathrm{cm}$, where the crosscorrelation analysis [7] fails. However, Table I shows that we can alternatively work with larger structures. Therefore, we have fabricated and studied microstructures with $H=5 \mu \mathrm{m}$, and the results will be presented in the next section.

\section{HIGH ELECTRIC FIELD: TWO MIGRATION STATES}

We begin by discussing the simulation results. We started with studying the extreme case $E=E_{0}$. This corresponds to $430 \mathrm{~V} / \mathrm{cm}$ in a $5 \mu \mathrm{m}$ structure and is thus not accessible in experiments. Two sample trajectories for a chain of length $N=10(3.4 \mathrm{kbp})$ and $N=400(140 \mathrm{kbp})$ monomers are given in Fig. 4. As can be seen from the insets in the $N=10$ case, the chains retain some memory of their state in the previous cavity. If a chain passes a constriction without being trapped, it will avoid trapping at the next barrier as well. A similar effect had already been observed in the entropic traps [15]. As in the simulations discussed in the previous section, chains may still get trapped in the corners of cavities, but at a)

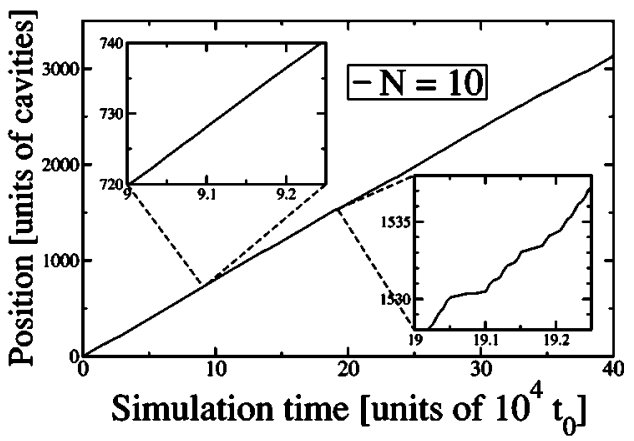

b)

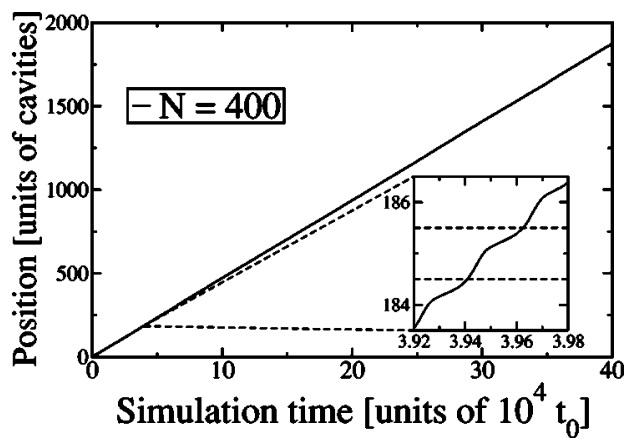

FIG. 4. Trajectories of a chain with (a) $N=10(3.4 \mathrm{kbp})$ and (b) $N=400(140 \mathrm{kbp})$ monomers at $E=E_{0}(430 \mathrm{~V} / \mathrm{cm})$. The dashed lines inside the inset in the $N=400$ case represent the onset of the narrow regions.

a lower rate due to the reduced diffusion time inside a single cavity. For $N=400$, the migration is periodically fast and slow, indicating that the polymer penetrates the low field regions in the cavities. Over long simulation times, both the trajectories for $N=10$ and $N=400$ are quite regular.

The situation is qualitatively different for $N=200$ (70 kbp). The trajectory is smooth on short timescales, but occasionally, the speed of migration changes abruptly (Fig. 5). One observes two states of migration, one of which is fast and the other is slow. The lower panel of Fig. 5 shows that the two states are associated with two distinctly different $z$ positions of the center of mass: the polymer migrates faster when it stays in the homogeneous field in the upper part of the channel. From Fig. 5, both states are long-lived during the simulation. We note that a simulation of a chain of length $N$ in a channel of height $H$ and the field $E$ is equivalent to a simulation of a chain of length $N \lambda$ in a channel of height $H \lambda^{\nu}$ and the field $E \lambda^{\nu+1}$, if the time is rescaled with the factor $\lambda^{2 \nu+1}$ (cf. Sec. II B). Hence it is possible to construct an "infinite chain length limit" $N \rightarrow \infty$ such that one has two migration states, and the timescale for transition between these states diverges. In this sense, we observe a nonequilibrium first-order phase transition between two coexisting phases (Fig. 8). The nonmonotonic chain length dependence of the mobility at $E=0.04 E_{0}$ is a consequence of that transition.

The experimental studies of $\lambda$-DNA and T2-DNA migrating in large structures support our simulation results. In structures characterized by $H \approx 5 \mu \mathrm{m}$, at electric fields ranging up to $100 \mathrm{~V} / \mathrm{cm}$, both $\lambda$ - and T2-DNA exhibit two distinct states of migration, which are illustrated in Fig. 6(a). In 


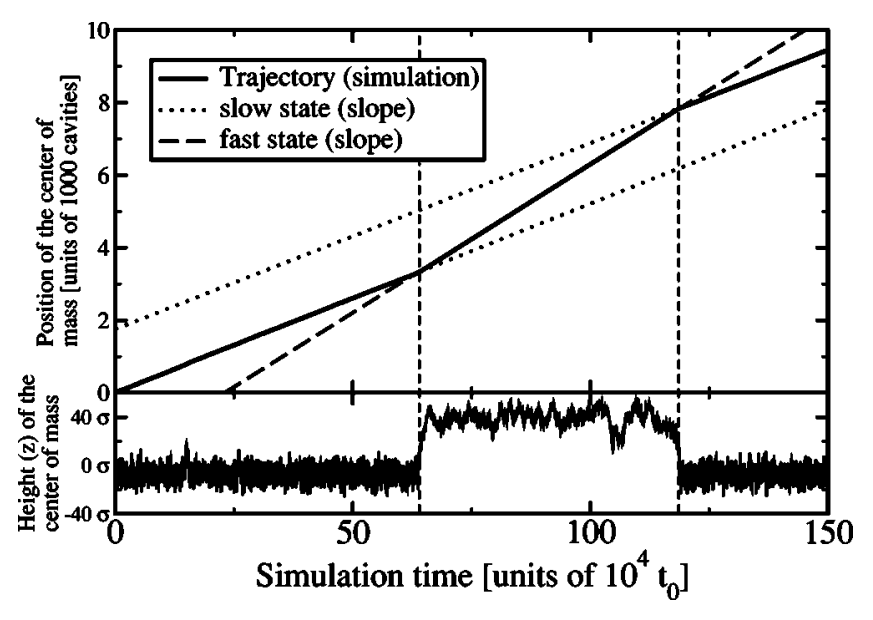

FIG. 5. Trajectory of a chain with $N=200(70 \mathrm{kbp})$ monomers at $E=E_{0}$, along the $x$ direction (top) and the $z$ direction (bottom). Note the two different migration speeds which can be related to the penetration depth into the wide cavities (the dashed vertical lines are just guides to the eye).

one state, the chain remains coiled all the time in the upper (homogeneous) part of the field, and travels at high speed. In the other, it penetrates deep into each cavity, forms a coil inside, and gets stretched again when moving into the narrow region.

a)

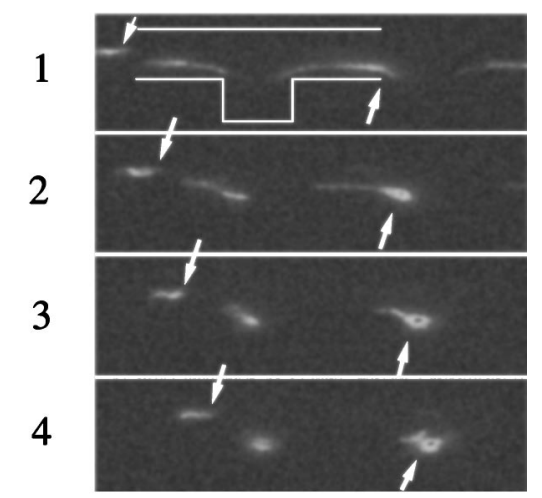

b)

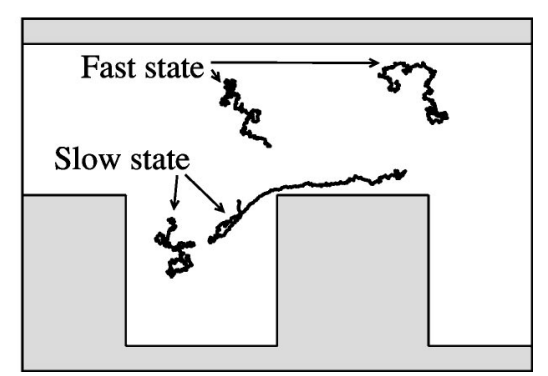

FIG. 6. Snapshots obtained from (a) an experiment with T2DNA and (b) simulation with 200 beads at $E=E_{0}$. The experimental snapshots show a series taken with $40 \mathrm{~ms}$ time step (fast state shown by arrows pointing down, slow state indicated by arrows pointing up). Both snapshots show chains in the slow state, which penetrate the wide region, form a coil inside and stretch when passing into the narrow region. Chains in the fast state are also shown. Here the polymer remains permanently coiled in the homogeneous part of the field. a)

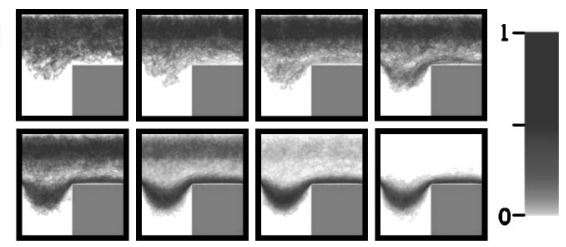

b)

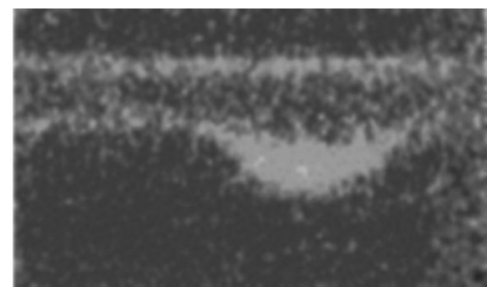

FIG. 7. (a) Monomer density histograms obtained from simulation for $N=50,100,120$, and 140 (top, left to right) and $N=170$, 200, 250, and 320 (bottom) at $E=E_{0}$. The density distribution $\rho$ was cut off at $\rho=1 / 2$ to dampen the peaks at the corners of the constrictions and to emphasize the depletion zone. (b) Monomer density histogram obtained from experiment at $E=86 \mathrm{~V} / \mathrm{cm}\left(0.20 E_{0}\right)$ for $\lambda$-DNA in $5 \mu \mathrm{m}$ constrictions. Note the depletion zone between both states.

Figure 7 shows monomer density histograms obtained from experiment and simulation. In both the experiments and in the simulations with chains of length $N=120-250$ beads, we observe a low-density depletion zone between the upper and the lower part of the channel. Furthermore, Fig. 7(a) shows that short chains are more likely to migrate in the fast state and long chains are more likely to migrate in the slow state. For $N=120-250$, the polymer migrates alternatingly in the slow and fast state, as has already been seen from the trajectory of $N=200$ in Fig. 5 .

Density histograms such as those shown in Fig. 7(a) allow to determine the population density of the two states. We choose the $z$ position of the center of mass as an indicator of a polymer's state (cf. also Fig. 5). Since the transition does not take place instantaneously (Fig. 9), we define two thresholds separated by a gap: a chain is taken to be in the slow state or in the fast state, if $z \leqslant 8 \sigma$ or $z \geqslant 20 \sigma$ over at least $5 \times 10^{6}$ time steps. After having assigned a state to large portions of the trajectory, we can determine the mobility in the slow and the fast state separately.

Figure 8(a) shows the results for $E=E_{0}$ and compares it with the overall mobility. The assignment of states works well for intermediate and long chains. For $N=100$ (34 kbp), the polymer migrates almost completely in the fast state and for polymers with $N \geqslant 320(110 \mathrm{kbp})$, the polymer occupies the slow state only. Short chains diffuse very strongly in the $z$ direction, no depletion zone occurs, and they often cross from the lower to the upper part of the channel. Therefore, the assignment criterion often fails, and only a few short fragments of the trajectory contribute to the calculation of the mobility in the fast state. This explains why the result does not coincide with the total mobility at low $N$.

The transition was not only observed at $E=E_{0}$, but also observed at smaller fields $E$. Figures 8(b) and 8(c) give the mobilities and the population densities for $E=0.25 E_{0}$ $(110 \mathrm{~V} / \mathrm{cm})$ and $E=0.04 E_{0}(17 \mathrm{~V} / \mathrm{cm})$, as determined by the 
a)

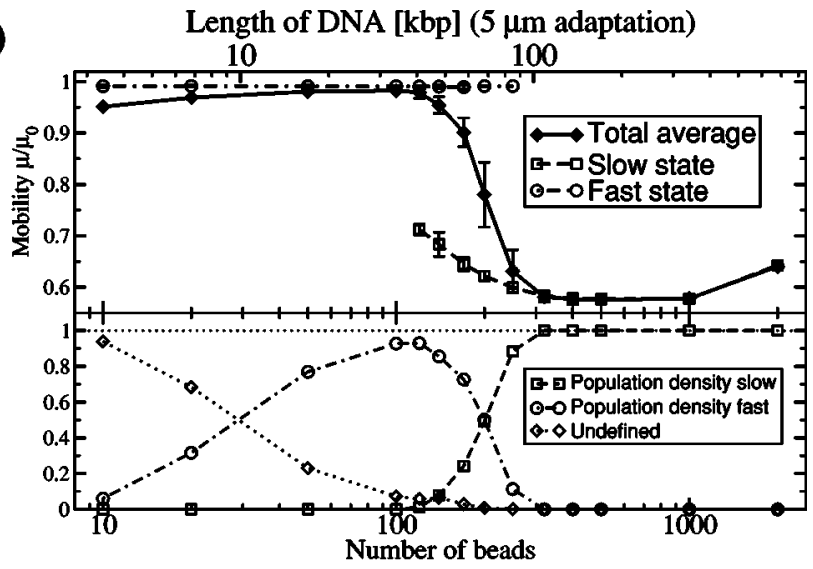

b)

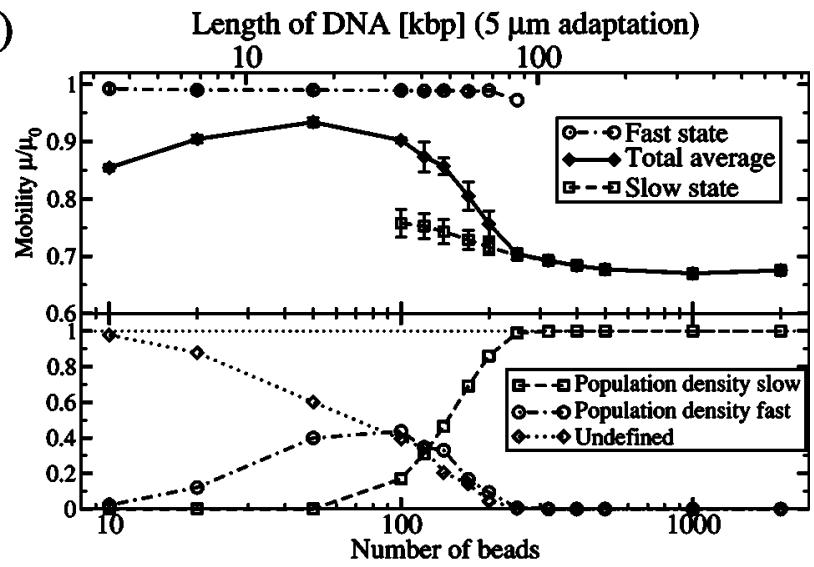

c)

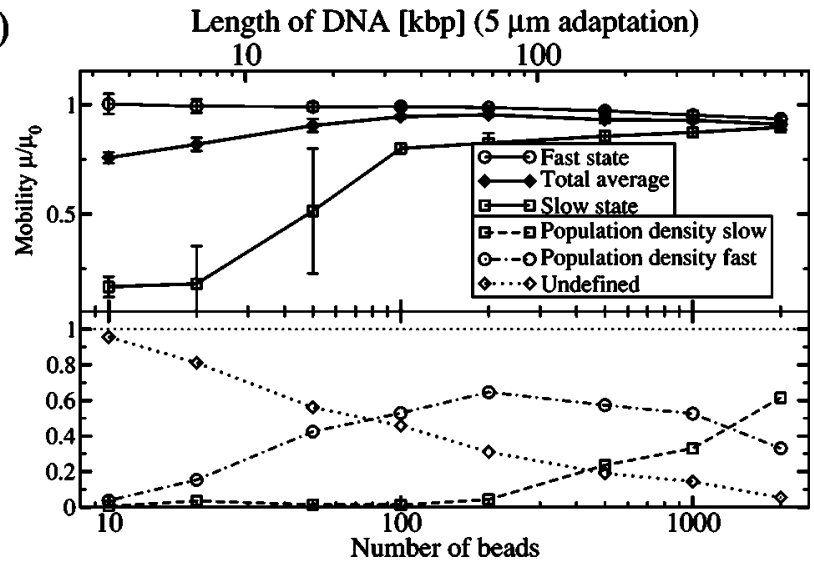

FIG. 8. Mobility as a function of the chains length at $E=E_{0}$ (a), $E=0.25 E_{0}$ (b), and $E=0.04 E_{0}$ (c). Note the decrease in mobility at $N=100-250(34-85 \mathrm{kbp})$ at $E=E_{0}$, which is also reflected by the population densities given in the lower panel. For short chains, diffusion dominates the migration, and most of the time, it is not possible to assign unambiguously a single migration state. This is reflected by the amount of "undefined" population. The error bars of the slow and fast mobilities show the statistical error, based on the spread of the values. Note that the transition fades away when the electric field is decreased.

above criterium. At $E=0.25 E_{0}$, the transition is not as pronounced as for $E=E_{0}$, but still present. At $E=0.04 E_{0}$, the transition has almost disappeared, and only a slight decrease in the overall mobility remains. This confirms our earlier

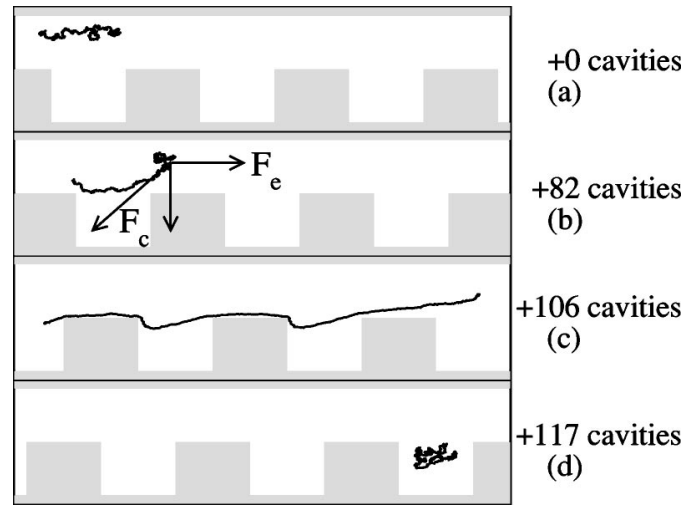

FIG. 9. Transition of chains with $N=500$ beads ( $170 \mathrm{kbp}$ ) from the fast to the slow state. The snapshots are shifted by the number of cavities given on the right. The transition itself takes about 35 cavities. In (b) a force parallelogram is sketched schematically. The electric force $F_{\mathrm{e}}$ and the entropic spring force $F_{\mathrm{c}}$ on the leading monomers add up to a total force which has a net downward component.

assertion that the nonmonotonic chain length dependence of the mobility at $E=0.04 E_{0}$ is a signature of the bistability. It also clarifies why long chains (T2-DNA) migrate slower than shorter chains $(\lambda$-DNA) in our channels. They tend to penetrate deeper into the low field regions, which slows them down significantly.

The quantitative comparison between simulations and experiments raises a question. According to the simulations, coexistence of two states should be observed at chain lengths below $N=250$ beads, which corresponds to $64 \mathrm{kbp}$ in $5 \mu \mathrm{m}$ microchannels. Thus we would expect two mobilities for $\lambda$-DNA, but only one for T2-DNA. Experimentally, we find that T2-DNA exhibits two states of migration as well. This is in contrast to the prediction of our simulation. However, a closer inspection reveals that even chains of $N=500$ beads (170 kbp, roughly T2-DNA) remain in a fast state for a long time (up to $8 \times 10^{8}$ time steps, data not shown), if they are prepared accordingly. These initial fast states turned out to be so stable, that we were unable to analyze their lifetime in detail. The experimental situation hardly corresponds to an "equilibrated" late-time limit. It is conceivable that after being introduced into the microfluidic channel, a sizable fraction of molecules happens to be prepared in a state that is very weakly populated in the long-time average.

\section{MIGRATION STATE TRANSITION MECHANISMS}

To understand the transition and identify the factors which stabilize the two migration states, we have investigated the transition processes between the two states in more detail.

\section{A. Transition from the fast to the slow state}

We begin with discussing the transition from the fast to the slow state. A series of snapshots of a chain with $N$ $=500$ beads in the process of crossing from the fast to the slow state is shown in Fig. 9. The chain travels through 
roughly 35 cavities during the transition process, which we can divide into five stages.

(1) At the beginning, the polymer is in a coiled conformation in the homogeneous field region. The radius of gyration of a chain of $N=500$ beads in free solution is $R_{\mathrm{g}} \approx 18 \sigma$. The size of the homogeneous field region is limited, thus larger polymers are deformed [Fig. 9(a)].

(2) At some stage, one polymer loop or end happens to penetrate into a region with a lower field. As that polymer part experiences a lower pulling force, it slows down, while the force acting on the rest of the polymer is unchanged. This leads to uncoiling and stretching of the polymer.

(3) More and more monomers are slowed down. Since the field in the homogeneous part pulls horizontally in the $x$ direction, and the delayed monomers are located in the cavities at low $z$ values, the total force acting on the chain has a net downward component [Fig. 9(b)]. After awhile, the polymer is completely stretched [Fig. 9(c)].

(4) For the highly stretched chain [Fig. 9(c)], the probability that the foremost monomer gets trapped in a cavity is very high. Immediately afterwards, the polymer collapses in that cavity [Fig. 9(d)].

(5) At the end, the polymer migrates in the slow state. For long polymers, the probability to leave the slow state is very low [Fig. 9(d)].

\section{B. Transition from the slow to the fast state}

Unfortunately, the configuration snapshots of the transition from the slow to the fast state cannot be interpreted as easily as those in Fig. 9, and we have not yet been able to distil the mechanisms which stabilize the fast state in a satisfactory way.

One effect which presumably favors the transtion is the inertia of the molecule at the corners of the constriction. Monomers migrating up at high speed against the wall continue to drift upwards slightly as they enter the constriction (data not shown). This effect involves dynamical processes on timescales smaller than $\tau_{0}$, the characteristic decay time for the drift velocity of the polymer. In our model, $\tau_{0}$ is given by $\tau_{0}=m / \zeta$, which corresponds to approximately $10^{-4} \mathrm{~s}$ for beads of mass $m=\zeta t_{0}$. For DNA in free solution, the drift velocity decay time is [29] $\tau_{0} \approx 10^{-9} \ldots 10^{-12} \mathrm{~s}$, which is much smaller. Thus this particular drift effect is an artifact of the simulation model. To verify that this mechanism is not responsible for the transition observed in the simulations, we modified the model by changing its dynamics to that of an overdamped system [30] [Eq. (7)]. We could only simulate short chains, and were unable to perform a thorough analysis due to the reduced time step [Eq. (7)]. The transition still occurs in both directions (data not shown). This proves that the fast state is stabilized by another mechanism. In fact, the only noticeable effect of inertia was to suppress the transition for short chains.

Another conceivable mechanism which might favor and stabilize the fast state is based on diffusion. While the polymer migrates along the wall in the narrow region and around the corners, it can only diffuse upwards. This effect is enhanced by the tendency of the polymer, which has been
TABLE II. Crossover lengths $N_{\mathrm{c}}$ for various electric fields. Errors of the crossover lengths are estimates of the population density histograms.

\begin{tabular}{llrc}
\hline \hline$E / E_{0}$ & \multicolumn{1}{c}{$N_{c}$} & $N_{c}\left(E / E_{0}\right)^{5 / 3}$ & $N_{c}\left(E / E_{0}\right)^{2 / 3}$ \\
\hline 0.08 & $290 \pm 25$ & $4.3 \pm 0.4$ & $55 \pm 5$ \\
0.15 & $174 \pm 20$ & $7.4 \pm 0.9$ & $50 \pm 6$ \\
0.25 & $124 \pm 15$ & $12.3 \pm 1.5$ & $50 \pm 6$ \\
0.35 & $120 \pm 15$ & $20.9 \pm 2.6$ & $60 \pm 8$ \\
0.50 & $106 \pm 10$ & $33.4 \pm 3.2$ & $67 \pm 6$ \\
0.70 & $125 \pm 10$ & $71.7 \pm 5.7$ & $103 \pm 8$ \\
1.00 & $200 \pm 5$ & $200.0 \pm 5.0$ & $200 \pm 5$ \\
\hline \hline
\end{tabular}

forced into a stretched conformation in the inhomogeneous parts of the field, to contract back to a coil. The characteristic timescale for contraction is presumably related to the Rouse time and scales like $N^{1+2 \nu}$ [20], which would explain why the slow state dominates at large chain length. We have not yet been able to support this explanation quantitatively.

The understanding of the fast state remains an open problem. Systematic studies are difficult, because the opposite transition is triggered by diffusion as well.

\section{Critical chain length}

Figure 9 suggests that the transition can only take place if the electric field exceeds a critical value; the inhomogeneous field has to uncoil the chain and thus work against the entropic spring force of the polymers. The mean elongation $\langle r\rangle$ of stretched self-avoiding polymers subject to a force $f$, which pulls on the end monomers, is [31]

$$
\langle r\rangle \propto N a\left(f a / k_{\mathrm{B}} T\right)^{2 / 3},
$$

for strongly stretched chains, and

$$
\langle r\rangle \propto a^{2} N^{2 v} /\left(k_{\mathrm{B}} T\right) f
$$

for weakly stretched chains, where $a \approx 0.5 \sigma$ is the proportionality constant in $R_{\mathrm{g}}=a N^{\nu}$. In our case, the force of course acts on all monomers. Nevertheless, we shall use Eqs. (10) and (11) to roughly estimate the maximal effect of the inhomogeneous field on a chain, with $f \propto E$.

The question is how much does the field have to stretch a chain in order to stabilize a distinct slow state? Let us consider the critical chain length $N_{\mathrm{c}}$ at which both states are equally populated. Assuming that it is sufficient to stretch the chains by an amount proportional to their own size, one would expect $N E^{5 / 3}$ to be a constant at $N=N_{\mathrm{c}}$ [according to both Eqs. (10) and (11)]. Alternatively, it might be necessary to stretch the chain to a fixed length, which is determined by the size of the device, in particular the constriction length $H$ [see Fig. 1(b)]. In that case, according to Eq. (10), $N E^{2 / 3}$ should be constant.

This is tested in Table II. The quantity $N_{\mathrm{c}} E^{5 / 3}$ is obviously not constant for different electric fields $E$. However, $N_{\mathrm{c}} E^{2 / 3}$ is almost constant for $E \leqslant 0.50 E_{0}$. For larger values of $E$ this rule breaks down. This might be related to the inertia effects discussed in Sec. VI B. Simulations with overdamped dy- 
namics at $E=E_{0}$ indeed suggest that, as $m \rightarrow 0$, chains as small as $N=50$ already exhibit two distinct migration speeds (data not shown). This would yield $N_{\mathrm{c}} E^{2 / 3}=50 E_{0}^{2 / 3}$, which is consistent with results at lower fields. Unfortunately, the statistical quality of our data is not sufficient to determine $N_{\mathrm{c}}$ rigorously in the overdamped case.

Nevertheless, the sum of our findings suggests that the critical length is determined by the geometry of the device rather than by the size of the chain. This could explain why the transition disappears at small fields: for $E=0.04 E_{0}$, the predicted critical length is $N_{\mathrm{c}} \approx 500$, but free chains of that length have an end-to-end distance of $46 \sigma$, which is comparable to the channel height $H$. Hence a fast state, in which the chain is essentially unperturbed, cannot exist.

This result suggests strategies for the design of microstructures which do or do not exhibit the two-state behavior; we expect it can be suppressed by choosing the constriction length $d$ in Fig. 1(b) small, and it can be promoted by making $d$ larger.

\section{CONCLUSION}

We have presented Brownian dynamics simulations and experimental studies of DNA migration in structured microchannels. Our simulations reproduce earlier experimental results presented by Duong et al. [7] and, in particular, explain the experimentally observed migration order of $\lambda$ - and T2DNA in migration studies and separation experiments [7]: in channels with geometries as shown in Fig. 1, and for moderate field values, the shorter $\lambda$-DNA molecules migrate faster than longer T2-DNA molecules.

This behavior, which is opposite to that expected at weaker fields, is a signature of a high-field nonequilibrium bistability. At very high fields (or in much larger structures), we found that chains can migrate with two distinctly different speeds, assuming different states of migration. This was observed both experimentally and in simulations. We have discussed the factors which stabilize the two states and can thus propose strategies for the design of channels which do or do not exhibit the transition, and which could be suitable for separation experiments.

The comparison of simulations and experiments demonstrates that our simple DNA model, which disregards electrostatic interactions, hydrodynamic interactions, and irrota- tional fluid flow, nevertheless reproduces the experimental results of DNA migration in our microchannels. However, the interplay of DNA motion and buffer flow is nontrivial and may be neglected for irrotational EOF outside Debye layers only. As mentioned in Sec. II B, the experimental electro-osmotic mobility in straight channels is of the same order of magnitude as the DNA mobility. In structured microchannels, where DNA molecules migrate at either high fields or along the Debye layer, electroosmosis should give rise to interesting nontrivial flow patterns which certainly influence the DNA migration. Moreover, the electrostatic and the hydrodynamic interactions are not entirely screened in microchannels, which leads to additional effects [26]. In order to investigate such phenomena, systematic experimental studies are necessary, and efficient new simulation methods need to be developed. These shall be explored in the future.

From a practical point of view, our results have two important implications.

On the one hand, they demonstrate that the physics of DNA electrophoresis in microchannels is surprisingly complex. When designing new microfluidic devices, one must be aware of the possibility of nonmonotonic or even bistable behavior, and a thorough theoretical analysis is advisable.

On the other hand, the observed two-state behavior can presumably be exploited. Our findings indicate that the migration velocities of the DNA fragments differ by a large factor (almost 2 in Fig. 8) for $N>N_{\mathrm{c}}$ and $N<N_{\mathrm{c}}$. Since the chain length corresponding to $N_{\mathrm{c}}$ depends on the channel geometry (cf. Table I), a device with a gradient in the constriction length could in principle sequentially separate molecules very efficiently. However, as the switching time between the two states is very long, methods need to be developed to accelerate the transition. We are currently exploring corresponding strategies experimentally as well as theoretically.

\section{ACKNOWLEDGMENTS}

We would like to thank Ralf Eichhorn for carefully reading the manuscript. This work was funded by the German Science Foundation (SFB 613, Teilprojekt D2). Parts of the simulation jobs were handled by the job queuing system Condor, which was developed by the Condor Team at the Computer Science Department of the University of Wiscon$\sin [32]$.
[1] J.-L. Viovy, Rev. Mod. Phys. 72, 813 (2000).

[2] C. S. Effenhauser, G. J. M. Bruin, and A. Paulus, Electrophoresis 18, 2203 (1997).

[3] D. Schmalzing, L. Koutny, A. Adourian, P. Belgrader, P. Matsudaira, and D. Ehrlich, Proc. Natl. Acad. Sci. U.S.A. 94, 10273 (1997).

[4] S. W. Turner, A. M. Perez, A. Lopez, and H. G. Craighead, J. Vac. Sci. Technol. B 16, 3835 (1998).

[5] H.-P. Chou, C. Spence, A. Scherer, and S. Quake, Proc. Natl. Acad. Sci. U.S.A. 96, 11 (1999).
[6] J. Han and H. G. Craighead, Science 288, 1026 (2000).

[7] T. T. Duong, R. Ros, M. Streek, F. Schmid, J. Brugger, D. Anselmetti, and A. Ros, Microelectron. Eng. 67-68, 905 (2003).

[8] O. Bakajin, T. A. J. Duke, J. Tegenfeldt, C.-F. Chou, S. S. Chan, R. H. Austin, and E. C. Cox, Anal. Chem. 73, 6053 (2001).

[9] L. R. Huang, J. O. Tegenfeldt, J. J. Kraeft, J. C. Sturm, R. H. Austin, and E. C. Cox, Nat. Biotechnol. 20, 1048 (2002).

[10] A. Ros, W. Hellmich, T. Duong, and D. Anselmetti, J. Biotech- 
nol. 112, 65 (2004).

[11] J. Han, S. W. Turner, and H. G. Craighead, Phys. Rev. Lett. 83, 1688 (1999); 86, 1394(E) (2001).

[12] J. Han and H. G. Craighead, Anal. Chem. 74, 394 (2002).

[13] F. Tessier, J. Labrie, and G. W. Slater, Macromolecules 35, 4791 (2002).

[14] Z. Chen and F. A. Escobedo, Mol. Simul. 29, 417 (2003).

[15] M. Streek, F. Schmid, T. T. Duong, and A. Ros, J. Biotechnol. 112, 79 (2004).

[16] J. M. Deutsch, Phys. Rev. Lett. 59, 1255 (1987).

[17] J. M. Deutsch, Science 240, 922 (1988).

[18] M. Matsumoto and M. Doi, Mol. Simul. 12, 219 (1994).

[19] H. Noguchi and M. Takasu, J. Chem. Phys. 114, 7260 (2001).

[20] M. Doi and S. F. Edwards, The Theory of Polymer Dynamics (Oxford Science, New York, 1986).

[21] B. Dünweg and W. Paul, Int. J. Mod. Phys. C 2, 817 (1991).

[22] M. Streek, "Migration of dna on a structured surface in an external field," diploma thesis, Universität Bielefeld, 2002.

[23] D. E. Smith, T. T. Perkins, and S. Chu, Phys. Rev. Lett. 75, 4146 (1995).
[24] E. B. Cummings, S. K. Griffiths, R. H. Nilson, and P. H. Paul, Anal. Chem. 72, 2526 (2000).

[25] J. G. Santiago, Anal. Chem. 73, 2353 (2001).

[26] D. Long, J.-L. Viovy, and A. Ajdari, Phys. Rev. Lett. 76, 3858 (1996).

[27] E. Stellwagen, Y. Lu, and N. C. Stellwagen, Biochemistry 42, 11745 (2003).

[28] X. Huang, M. J. Gordon, and R. N. Zare, Anal. Chem. 60, 1837 (1988).

[29] P. D. Grossmann and J. C. Colburn, Capillary Electrophoresis, Theory and Practice: Free Solution Capillary Electrophoresis (Academic, San Diego, 1992).

[30] P. Reimann, Phys. Rep. 361, 57 (2002).

[31] A. Baumgärtner, in Soft Matter-Complex Materials on Mesoscopic Scales, edited by J. K. G. Dhont, G. Gompper, and D. Richter (33. IFF-Ferienkurs, Forschungszentrum Jülich, 2002), p. B3.

[32] The Condor Team, 2003. Software package from www.cs.wisc.edu/condor/ 